\title{
DERIVATIONS OF HIGHER ORDER IN SEMIPRIME RINGS
}

\author{
JIANG LUH \\ Department of Mathematics \\ North Carolina State University \\ Raleigh, NC 27695-8205 U.S.A. \\ YOUPEI YE \\ Department of Computer Science \\ Nanjing University of Science and Technology \\ Nanjing, China \\ (Received September 12, 1996 and in revised for April 2, 1997)
}

\begin{abstract}
Let $R$ be a 2-torsion free semiprime ring with derivation $d$. Supposed $d^{2 n}$ is a derivation of $R$, where $n$ is a positive integer. It is shown that if $R$ is $(4 n-2)$-torsion free or if $R$ is an inner derivation of $R$, then $d^{2 n-1}=0$.
\end{abstract}

KEY WORDS AND PHRASES: Derivation, semiprime ring, nilpotent derivation. 1992 AMS SUBJECT CLASSIFICATION CODES: 16 W25; 16 N60.

Recently, the present authors in [5] showed that, if $d$ is a derivation of a prime ring of characteristic $\neq 2$ and if $d^{2 n}$ is also a derivation of $R$, then $d^{2 n-1}=0$. This generalizes several earlier results given by Posner, Martindale, Miers, Jensen, Chung and Luh $[1,2,3,4,5]$. The purpose of this paper is to extend this result to semiprime rings. More precisely, we prove the following two theorems.

THEOREM 1. Let $R$ be a semiprime ring with derivation $d$. If $d^{2 n}$ is a derivation and if $R$ is $(4 n-2)$ !-torsion free, then $d^{2 n-1}=0$.

THEOREM 2. Let $R$ be a 2-torsion free semiprime ring and $d$ an inner derivation of $R$. If $d^{2 n}$ is a derivation, then $d^{2 n-1}=0$.

The following three lemmas will be used in the proof of the theorems. From the nature of the result one would expect the first lemma to be known, but we have not been able to locate it in the literature.

LEMMA 1. Suppose $R$ is an $m$-torsion free semiprime ring. Then there are prime ideals 
$P_{i}$ of $R, i \in B$, such that $\bigcap_{i \in B} P_{i}=(0)$, and the characteristic of each $R / P_{i}$ does not divide $m$.

PROOF. Since $R$ is semiprime, $R$ is a subdirect sum of prime rings. That is, there are prime ideals $P_{i}$ of $R, i \in A$, such that $\bigcap_{i \in A} P_{i}=(0)$. Let $B=\left\{i \in A: \operatorname{char} R / P_{i}\right.$ does not divide $m\}$. We need to show that $\bigcap_{i \in B} P_{i}=(0)$. Let $x \in \bigcap_{i \in B} P_{i}$. Clearly, $m x \in P_{i}$ for all $i \in B$. Furthermore, for $i \in A \backslash B$, since $\operatorname{char} R / P_{i}$ divides $m, m x \in P_{i}$. Therefore, $m x \in \bigcap_{i \in A} P_{i}$ and consequently $m x=0$. Since $R$ is $m$-torsion free, $x=0$ and hence $\bigcap_{i \in B} P_{i}=(0)$ as we desired.

LEMMA 2. Let $R$ be a ring with derivation $d$ and $P$ a prime ideal of $R$. Suppose $d^{2 n}$ is a derivation and the characteristic of $R / P$ does not divide $2 n$. If $a \in R$ and $s$ is the least positive integer such that $d^{s}(a) \notin P$, then $s<2 n-1$.

PROOF. From Leibniz' rule, for any $x, y \in R$,

$$
\begin{aligned}
& d^{2 n}(x y)=\sum_{i=0}^{2 n}\left(\begin{array}{c}
2 n \\
i
\end{array}\right) d^{2 n-i}(x) d^{i}(y) \quad \text { and } \\
& d^{2 n}(x y)=d^{2 n}(x) y+x d^{2 n}(y),
\end{aligned}
$$

which imply that

$$
\sum_{i=1}^{2 n-1}\left(\begin{array}{c}
2 n \\
i
\end{array}\right) d^{2 n-i}(x) d^{i}(y)=0 .
$$

Suppose, to the contrary, that $s \geq 2 n-1$. In (1), replace $x$ by $d^{s-2 n+1}(a)$ and note that $d^{j}(a) \in P$ for all $j<s$. We obtain

$$
2 n d^{s}(a) d(y) \in P \text { for all } y \in R
$$

Replacing $y$ by $x y$ in (2) yields

$$
2 n d^{s}(a) x d(y)+2 n d^{s}(a) d(x) y \in P \text { for all } x, y \in R .
$$

From (2) and the primeness of $P$, it follows that $2 n d^{s}(a) \in P$.

Since char $R / P$ and $2 n$ are relatively prime, $d^{s}(a) \in P$, a contradiction. Hence $s<2 n-1$.

We should note that in Lemma 2 even if $a \in P, d(a)$ need not be in $P$. In view of this, we define

$$
P^{*}=\left\{x \in P: d^{k}(x) \in P \text { for all positive integers } k\right\} \text {. }
$$

It is easy to see that $P^{*}$ is an ideal of $R$ contained in $P$ and $d\left(P^{*}\right) \subset P^{*}$. If $d$ is inner, then $d(P) \subset P$ and $P^{*}=P$. In the next lemma, we will show that $P^{*}$ is a prime ideal under a restriction on the characteristic of $R / P$.

LEMMA 3. Let $R$ be a ring with derivation $d$ and $P$ a prime ideal of $R$. If $d^{2 n}$ is a derivation and if the characteristic of $R / P$ does not divide $(4 n-2) !$, then $P^{*}$ is a prime ideal of $R$. 
PROOF. Suppose not. Then there exist $a, b \in R$ such that $a R b \subset P^{*}$ but $a, b \notin P^{*}$. Since $a R b \subset P$ and $P$ is prime, either $a \in P$ or $b \in P$. First, we claim that $a$ and $b$ both belong to $P$. Suppose $b \notin P$, say. Then $a \in P$. Since $d(a x b) \in P$ or $d(a) x b+a d(x b) \in P$, it follows that $d(a) x b \in P$ for all $x \in R$. By the primeness of $P, d(a) \in P$. Now, since $d^{2}(a x b) \in P$ or $d^{2}(a) x b+2 d(a) d(x b)+a d(x b) \in P$, it follows that $d^{2}(a) x b \in P$ for all $x \in R$. Consequently, $d^{2}(a) \in P$. Continuing this process, we obtain that $d^{k}(a) \in P$ for all positive integers $k$, i.e. $a \in P^{*}$, a contradiction. Hence $a, b$ both lie in $P$. Next, let $s, t$ be the least positive integers such that $d^{s}(a) \notin P$ and $d^{t}(b) \notin P$. From $d^{s+t}(a x b) \in P$ and using Leibniz' rule, we obtain

$$
\sum_{j=0}^{s+t}\left(\begin{array}{c}
s+t \\
j
\end{array}\right) d^{j}(a x) d^{s+t-j}(b) \in P \text { for all } x \in R .
$$

By assumption, $d^{s+t-j}(b) \in P$ for $j>s$, and hence (3) becomes

$$
\sum_{j=0}^{s}\left(\begin{array}{c}
s+t \\
j
\end{array}\right) d^{j}(a x) d^{s+t-j}(b) \in P \quad \text { for all } x \in R .
$$

'For $j<s$, another application of Leibniz' rule yields

$$
d^{j}(a x)=\sum_{i=0}^{j}\left(\begin{array}{l}
j \\
i
\end{array}\right) d^{i}(a) d^{j-i}(x) \in P \text { for all } x \in R,
$$

since $d^{i}(a) \in P$, for all $i<s$. Hence (4) can be reduced further to

$$
\left(\begin{array}{c}
s+t \\
s
\end{array}\right) d^{s}(a x) d^{t}(b) \in P \text { for all } x \in R
$$

which finally by Leibniz' rule again gives

$$
\left(\begin{array}{c}
s+t \\
s
\end{array}\right) d^{s}(a) x d^{t}(b) \in P \text { for all } x \in R .
$$

Note that, since $s, t<2 n-1$ by Lemma $2, s+t<4 n-2$ and $\left(\begin{array}{c}s+t \\ s\end{array}\right)$ divides $(4 n-2)$ !. By our assumption that char $R / P$ does not divide $(4 n-2) !,(5)$ implies that

$$
d^{s}(a) x d^{t}(b) \in P \text { for all } x \in R .
$$

By the primeness of $P$, either $d^{s}(a) \in P$ or $d^{t}(b) \in P$. But this is absurd. Therefore, $P^{*}$ is a prime ideal.

PROOF OF THEOREM 1. By Lemma 1, there are prime ideals $P_{i}$ of $R, i \in B$, such that $\bigcap_{i \in B} P_{i}=(0)$, and $\operatorname{char} R / P_{i}>4 n-2$ for all $i \in B$. According to Lemma 3, $P_{i}^{*}$ is a prime ideal of $R$ contained in $P_{i}$ and $d\left(P_{i}^{*}\right) \subset P_{i}^{*}$, for each $i \in B$. Since $\bigcap_{i \in B} P_{i}=(0)$ and $P_{i}^{*} \subset P_{i}$ for each $i \in B$, it follows that $\bigcap_{i \in B} P_{i}^{*}=(0)$. Moreover, $d$ induces a derivation $d_{i}$ of $R / P_{i}^{*}$ and $d_{i}^{2 n}$ is a derivation of $R / P_{i}^{*}$. By a result in [5], $d_{i}^{2 n-1}=0$, or $d_{i}^{2 n-1}\left(x+P_{i}^{*}\right)=P_{i}^{*}$ for each $i \in B$ and for all $x \in R$. That is, $d^{2 n-1}(x) \in P_{i}^{*}$ for each $i \in B$ and for all $x \in R$. Consequently, $d^{2 n-1}(x)=0$ for all $x \in R$. Hence $d^{2 n-1}=0$ as we desired. This completes the proof. 
PROOF OF THEOREM 2. Since $R$ is 2-torsion free and semiprime, there are prime ideals $P_{i}$ of $R$ with char $R / P_{i} \neq 2$ for $i \in B$. Since $d$ is inner, $d\left(P_{i}\right) \subset P_{i}$ or $P_{i}^{*}=P_{i}$ for each $i \in B$. Using an argument similar to the proof of Theorem 1, but without using Lemma 3, we can show that $d^{2 n-1}=0$.

\section{REFERENCES}

[1] CHUNG, L. O. and LUH, J., Nilpotency of Derivations, Canad. Math. Bull. 26 (1983), 341-346.

[2] JENSEN, D. W., Derivations of a Prime Ring Which Satisfy a Polynomial Identity, Thesis, North Carolina State University, 1983.

[3] MARTINDALE III, W. S. and MIERS, C. R., On the Iterates of Derivations of Prime Rings, Pacific J. Math. 104 (1983), 179-190.

[4] POSNER, E. C., Derivations in Prime Rings, Proc. Amer. Math. Soc. 8 (1957), 1093 1100.

[5] YE, Y. and LUH, J., Derivations of Higher Order in Prime Rings, Canad. Math. Bull. 39 (1996), 376-384. 


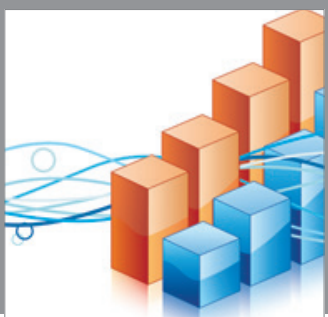

Advances in

Operations Research

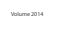

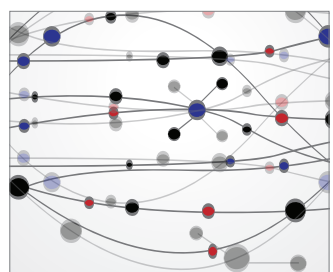

\section{The Scientific} World Journal
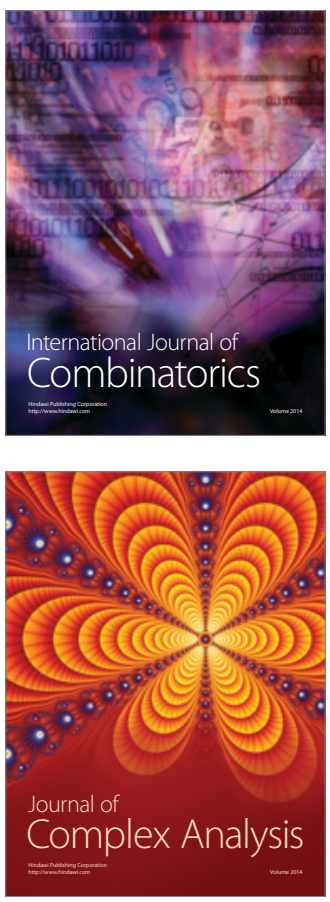

International Journal of

Mathematics and

Mathematical

Sciences
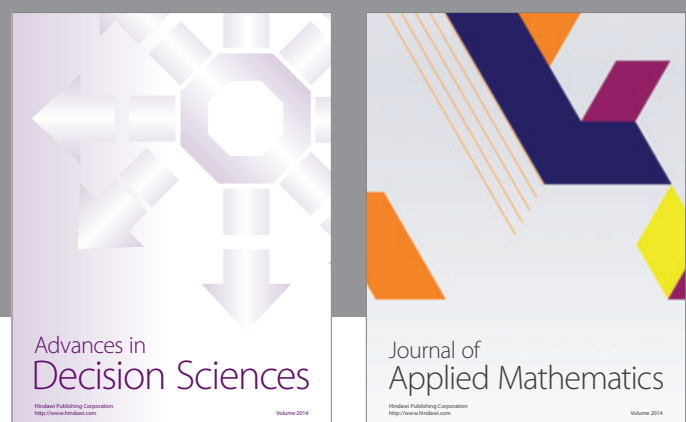

Journal of

Applied Mathematics
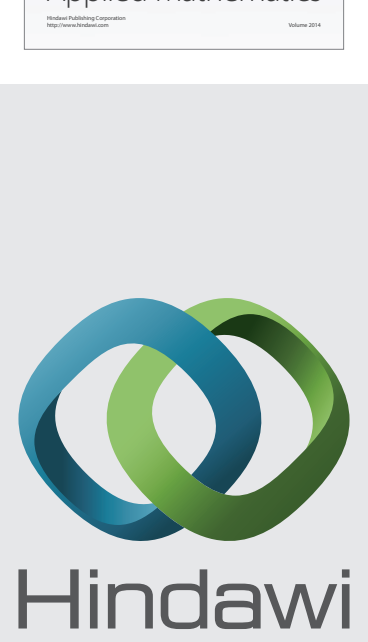

Submit your manuscripts at http://www.hindawi.com
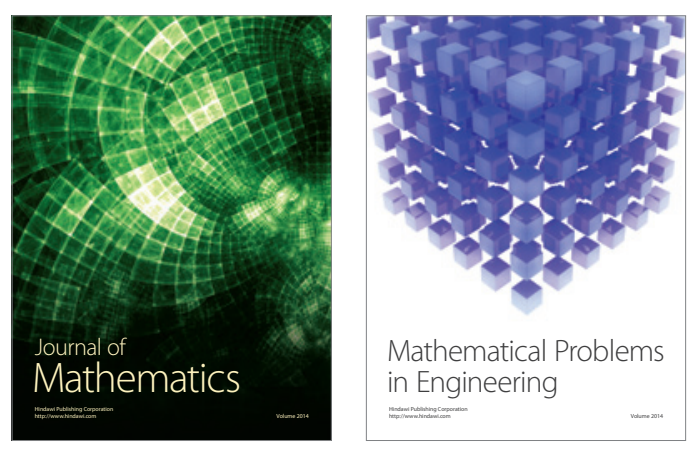

Mathematical Problems in Engineering
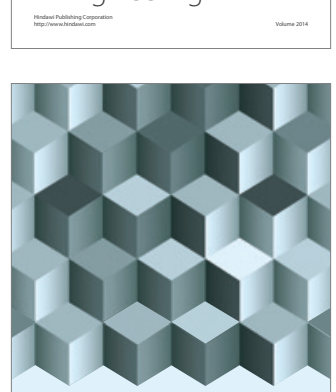

Journal of

Function Spaces
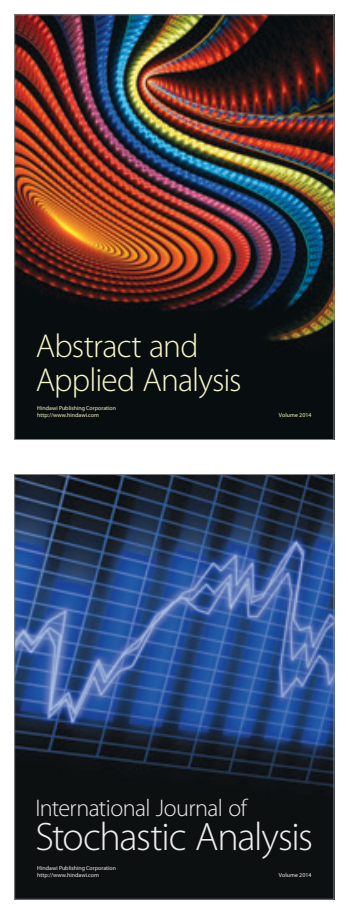

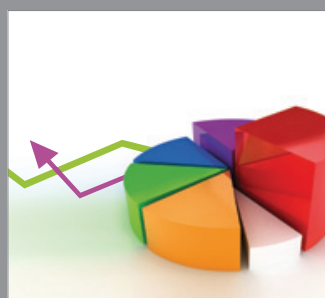

ournal of

Probability and Statistics

Promensencen
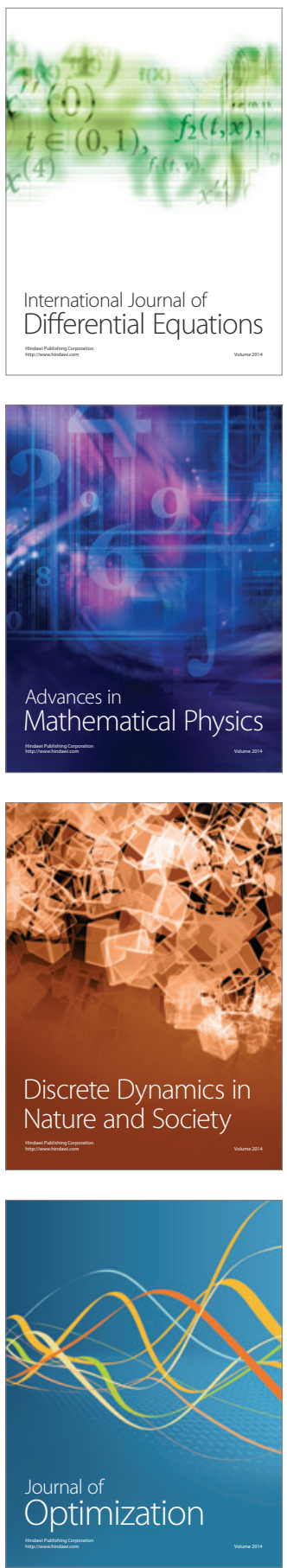